\title{
FROM FRACTAL ROBUSTNESS TO THE CRONE APPROACH
}

\author{
A. OUstaloup, J. SABATiER AND X. MOREAU
}

\begin{abstract}
This article deals with the transposition of "fractal robustness" to automatic control. The considered dynamic model which governs this phenomenon is a non integer order linear differential equation where the natural frequency and the damping ratio of the oscillatory mode of the solution are determined. A remarkable result is that the damping ratio is exclusively linked to the non integer order of the differential equation. This is the corner stone of the CRONE control. The dynamic behaviour of this control is described in tracking and in regulation. The robustness of the damping ratio and of the resonance ratio is demonstrated. An open loop is defined using closed loop performance specifications. A CRONE regulator is approximated by an integer order transmittance. Finally, the principle of the CRONE suspension, the synthesis method and the performance are developed.
\end{abstract}

RÉSumÉ. Cet article traite de la transposition en automatique de la "robustesse fractale". Le modèle dynamique considéré est une équation différentielle linéaire d'ordre non entier dont la fréquence propre et le facteur d'amortissement du mode oscillatoire de la solution sont déterminés. Un résultat remarquable tient à ce que le facteur d'amortissement est exclusivement lié à l'ordre non entier de l'équation différentielle. Un tel résultat est à l'origine de la commande CRONE. Le comportement dynamique de cette commande est décrit en asservissement et en régulation. La robustesse du facteur de résonance est démontrée. Un transfert en boucle ouverte est défini à partir des spécifications des performances en boucle fermée. Un régulateur CRONE est synthétisé par une transmittance d'ordre entier. Enfin, le principe de la suspension CRONE, sa méthode de synthèse et ses performances sont développés.

\section{INTRODUCTION}

The concept of fractal robustness may be illustrated by the relaxation of water on a porous dyke, where the damping ratio is independent of the mass of moving water [1]. This phenomenon is paradoxical in the integer approach to classical mechanics where the damping of any relaxation is dependent on the carried mass [2]. The example of relaxation of water on a porous dyke could be replaced by the relaxation of air on leaves or a lung, as leaves or lungs [3] also have a fractal dimension. The CRONE control or Contrôle Robuste d'Ordre Non Entier (which means non integer order robust control), where the degree of stability is robust with respect to the uncertainties of a plant [5], is based on this property.

In geometry, the continuity of the dimension is associated to the notion of fractality or of fractal [6].

In systemics, the continuity of the order of a system implies the notion of non integer order, more precisely non integer differentiation [7].

In other words whereas fractality comes under geometry, non integer differentiation comes under systemics, or more specifically system dynamics. The interdependence of these two concepts can only therefore appear when there is correlation

(C) Société de Mathématiques Appliquées et Industrielles. Typeset by LATEX.

A. Oustaloup, J. Sabatier, X. Moreau: Equipe CRONE - LAP - ENSERB Université Bordeaux I, 351, cours de la Libération - 33405 Talence cedex - France email : oustaloup@lap.u-bordeux.fr. 
between geometry and dynamics, or more specifically when geometry conditions a physical phenomenon ruled by a differential equation. Examples where the non integer derivative is used as a modelling tool are numerous in the various areas of physics [9] [10] [11] [12] etc ...

The paper is organized as follows.

From an observation by dyke builders on the relaxation of water on coastal breakwaters or river embankments whose damping is independent of the mass of moving water, a dynamic model governing the relaxation of water on a porous dyke is first given. The global model which results from the fundamental principle of dynamics proves to be remarkable both in its simplicity and its properties. It is a differential linear equation of non integer order between 1 and 2. In its solution the natural frequency of the relaxation depends on the mass of moving water. The damping ratio does not depend on this mass but on the non integer order of the differential equation dictated by the fractal dimension of the dyke.

The robustness of the damping is illustrated by two isodamping half-straight lines in the operational plane, and by a frequency template in the Nichols plane where the form and the vertical sliding ensure the invariance of the phase margin. The transposition of this template in automatic control defines the non integer approach used by the second generation CRONE control.

Then a description is given of the CRONE control's dynamic behavior in tracking and in regulation, and its frequential dynamic performances which translate in particular the robustness of the resonance ratio both in tracking and in regulation.

An open loop transfer including the template is described in the operational domain. Its description takes into account automatic control performance specifications : accuracy, dynamics and the sensitivity of the plant input. The parameters of this transfer are then determined.

The non integer order regulator deduced from this is then approximated by an integer order transmittance.

The usefulness of the non integer differentiation in vibration isolation is studied with the CRONE suspension. Performance obtained from a quarter-car model reveals, contrary to a traditional suspension where there is a interdependence massresonance and mass-damping, a great robustness of the resonance ratio and the damping ratio versus sprung mass variations ${ }^{1}$.

\section{Modeling of a robust natural Relaxation}

The aim of this section is to establish the dynamic model which governs the relaxation of water on a porous dyke.

Given a mass $M$ of water, the movement of which is its penetration into a permeable, or porous, dyke, and its velocity $V(t)$ (fig. 1), the application of the fundamental law of dynamics leads to the following differential equation :

$$
M \frac{d V(t)}{d t}+F(t)=0,
$$

in which $F(t)$ is the reaction force of the dyke, i.e. the resultant of the forces which solicit the water mass $M$.

If $S$ denotes the flowing section, speed $V(t)$ can be expressed as a function of $Q(t)$, namely :

\footnotetext{
${ }^{1}$ One of the technological solutions [24] developed for automotive domain was awarded the AFCET'95 trophy which distinguishes the best technological innovation stemming from University-Industry collaborations.

ESAIM: ProC., VOL. 5, 1998, 177-192
} 


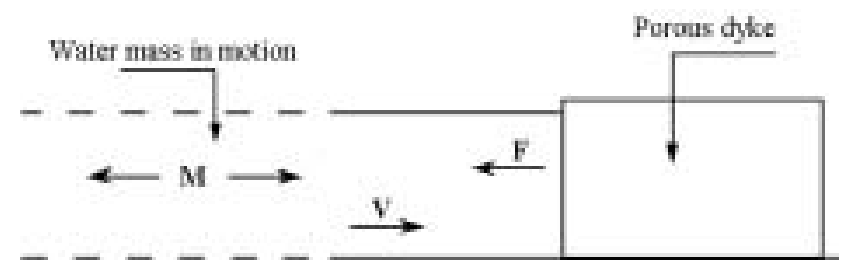

Figure 1. Study plant

$$
V(t)=Q(t) / S \text {. }
$$

Moreover, force $F(t)$ can be expressed as a function of the dynamic pressure $P(t)$ on the surface of the dyke, namely:

$$
F(t)=P(t) S .
$$

Using relations (2.2) and (2.3), one obtains a new form of the differential equation $(2.1)$ :

$$
\frac{M}{S^{2}} \frac{d Q(t)}{d t}+P(t)=0 .
$$

Considering the fractal character of the dyke porosity and the corresponding recursivity, it can be demonstrated that the water flow $Q(t)$ is proportional to the non integer derivative of the pressure $P(t)$ at the water-dyke interface, namely [1]:

$$
Q(t)=\frac{1}{\omega_{0}^{\alpha-1}}\left(\frac{d}{d t}\right)^{\alpha-1} P(t) \quad \text { with } \quad 1<\alpha<2,
$$

an equation which represents the dynamic model of the water dyke-interface. This proof is carried out through two different methods. The first uses a hypergeometric function [1]. The second is based on the smoothing of the Bode asymptotic diagrams of an equivalent electric admittance [16].

Including (2.5) in (2.4) provides a linear differential equation of non integer order $\alpha$ between 1 and 2 , namely :

$$
\frac{M}{S^{2}} \frac{1}{\omega_{0}^{\alpha-1}}\left(\frac{d}{d t}\right)^{\alpha} P(t)+P(t)=0
$$

or, under a canonical form :

$$
\tau^{\alpha}\left(\frac{d}{d t}\right)^{\alpha} P(t)+P(t)=0
$$

where $\tau$ denotes the transitional time constant defined by :

$$
\tau=\left(\frac{M}{S^{2}} \frac{1}{\omega_{0}^{\alpha-1}}\right)^{1 / \alpha} .
$$

\section{Fractal robustness}

The purpose of this section is to demonstrate the robustness of the damping ratio of the relaxation governed by (2.7), this robustness being called fractal robustness [13]. 


\subsection{ISODAMPING HALF-STRAIGHT LINES}

The characteristic equation of differential equation (2.7) is

$$
1+(\tau s)^{\alpha}=0
$$

namely :

$$
(\tau s)^{\alpha}=-1=e^{ \pm j \pi},
$$

the argument of -1 thus chosen (namely $\pm \pi$ ) being in accordance with the determination $]-\pi,+\pi$ [ of the argument of $s$ imposed by the undefined character of $s^{\alpha}$ for $s \in R^{-}$.

Such an equation admits no other than two poles, namely :

$$
s=\frac{1}{\tau} e^{ \pm j \pi / \alpha} ;
$$

for $1<\alpha<2$, their argument is indeed in accordance with the determination ]$-\pi,+\pi[$. The poles are complex and conjugated and form a centre angle $2 \Theta$ with $\Theta=(\pi-\pi / \alpha)$ (fig. 2). $\tau$ depending on $\mathrm{M}$, the poles move at a constant angle (fixed by the order $\alpha$ ) when M varies. The robustness in plane $s$ is then illustrated by two half-straight lines which form the same angle $\Theta$ in relation to the real axis and are called isodamping half-straight lines.

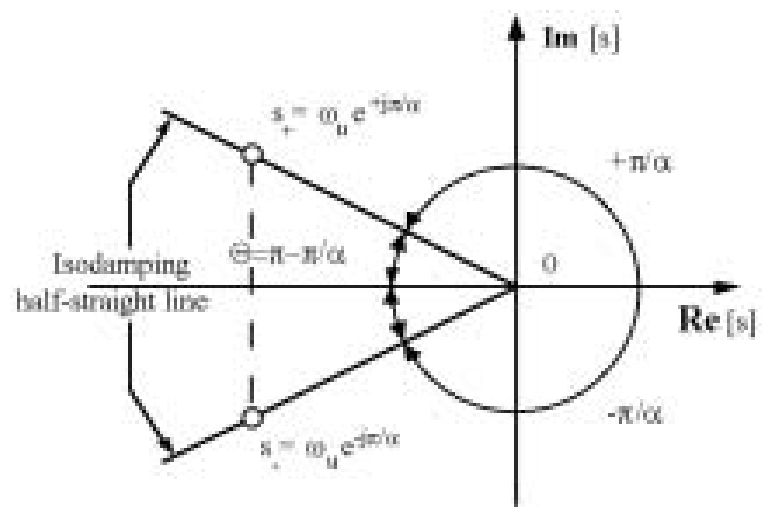

FigURE 2. Illustration of the robustness in the operational plane: the displacement of the roots on the isodamping half-straight line ensure the robustness of the damping

The natural frequency and the damping ratio are directly deduced from the poles, through their modulus $1 / \tau$ and the half-centre angle $\Theta$ that they form :

$$
\omega_{p}=\frac{1}{\tau} \sin \Theta=\frac{1}{\tau} \sin \left(\pi-\frac{\pi}{\alpha}\right)=\frac{1}{\tau} \sin \left(\frac{\pi}{\alpha}\right)
$$

and

$$
\zeta(\alpha)=\cos \Theta=\cos \left(\pi-\frac{\pi}{\alpha}\right)=-\cos \left(\frac{\pi}{\alpha}\right) .
$$

This result shows that the damping ratio $\zeta$ is exclusively a function of order $\alpha$, thus allowing the introduction of the notion of robust oscillatory mode. we summarize: 
The notion of robust oscillatory mode characterizes the concept of fractal robustness

which defines the robustness of the damping of natural phenomena, that fractality ensures through non integer differentiation.

\subsection{FREQUENCY TEMPlate}

Using the Laplace transform on (2.7), one obtains :

$$
(\tau s)^{\alpha} P(s)+P(s)=0,
$$

thus :

$$
P(s)=-\left(\frac{1}{\tau s}\right)^{\alpha} P(s) .
$$

This operational equation is translated by the functional diagram, fig. 3, which is the same as that of a free control (nil input $E(s)$ ). Because of a unit feedback, the direct chain determines an open loop transmittance of the form :

$$
\beta(s)=\left(\frac{1}{\tau s}\right)^{\alpha}=\left(\frac{\omega_{u}}{s}\right)^{\alpha},
$$

which is the transmittance of a non integer integrator in which $\omega_{u}=1 / \tau$ denotes the unit gain (or transitional) frequency.

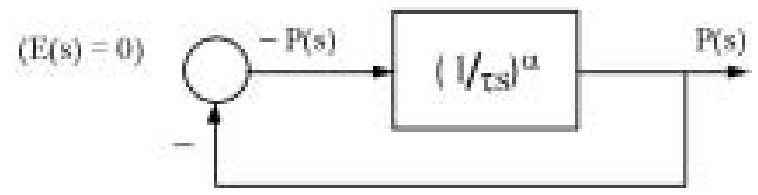

Figure 3. Functional diagram defining an open loop transfer

As $\arg \beta(j \omega)=-\alpha \pi / 2$ with $1<\alpha<2$, the Nichols locus [4] of $\beta(j \omega)$ is a vertical straight line of abscissa between $-\pi / 2$ and $-\pi$.

When $M$ changes, frequency $\omega_{u}$ is modified in conformity with the expression

$$
\omega_{u}=\frac{1}{\tau}=\left(\omega_{0}^{\alpha-1} \frac{S^{2}}{M}\right)^{1 / \alpha} .
$$

So, the straight line thus obtained slides on itself at the time of a variation of M. Such a vertical displacement ensures the constancy of the phase margin $\Phi_{m}$ [4], and thus the constancy of the corresponding damping ratio in the time domain, so translating the robustness of the damping.

In automatic control, the aim is to obtain a similar frequency behavior, in a medium frequency range around $\omega_{u}$, knowing that the closed loop dynamic behavior is exclusively linked to the open loop behavior around $\omega_{u}$ frequency.

This involves :

- an open loop Nichols locus which forms a vertical straight line segment around $\omega_{u}$ for the nominal parametric state of the plant, called open loop frequency template (or more simply template) (fig. 4) ; 


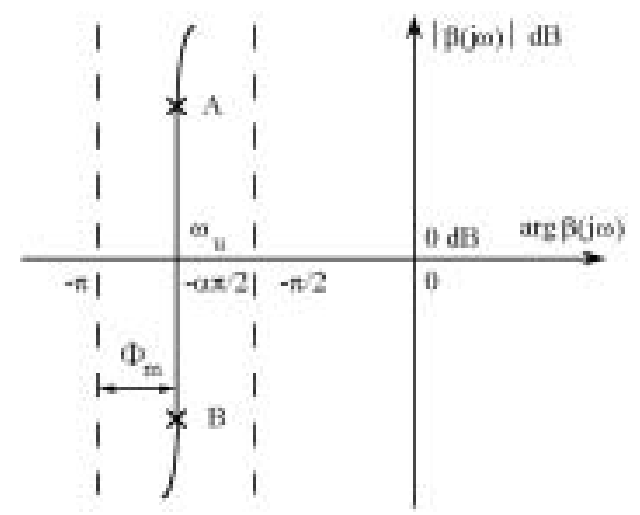

Figure 4. Illustration of robustness in the Nichols plane: the form and the vertical sliding of template $A B$ ensure the robustness of the damping through a constant phase margin $\phi_{m}$

- and a sliding of the template on itself at the time of a reparametration of the plant (this condition being verified when the reparametration of the plant only leads to gain variations around $\omega_{u}$ ).

Synthesizing such a template defines the non integer approach that the second generation CRONE control uses.

\section{Second generation CRONE control}

\subsection{Dynamic Behavior in Closed loop}

Given that the open loop behavior around the unit gain frequency determines the dynamic behavior in closed loop, the study of this behavior can be based on the transmittances in tracking $\mathbb{T}(s)$ and in regulation $\mathbb{S}(s)$ determined from the transmittance in open loop reduced to the transmittance of description of the template, $\boldsymbol{\beta}(s)$, namely :

$$
\beta(s)=\boldsymbol{\beta}(s) \quad \text { with } \quad \omega \in\left[\omega_{A}, \omega_{B}\right] \text {, }
$$

and

$$
\boldsymbol{\beta}(s)=\left(\frac{\omega_{u}}{s}\right)^{\alpha}, \quad \alpha \in[1,2] .
$$

$\mathbb{T}(s)$ and $\mathbb{S}(s)$ can be expressed as (fig. 5$)$ :

$$
\mathbb{T}(s)=\left[\frac{S(s)}{E(s)}\right]_{D(s)=0}=\frac{\boldsymbol{\beta}(s)}{1+\boldsymbol{\beta}(s)}=\frac{1}{1+\left(\frac{s}{\omega_{u}}\right)^{\alpha}}
$$

and

$$
\mathbb{S}(s)=\left[\frac{S(s)}{D(s)}\right]_{E(s)=0}=\frac{1}{1+\boldsymbol{\beta}(s)}=\frac{\left(\frac{s}{\omega_{u}}\right)^{\alpha}}{1+\left(\frac{s}{\omega_{u}}\right)^{\alpha}} .
$$




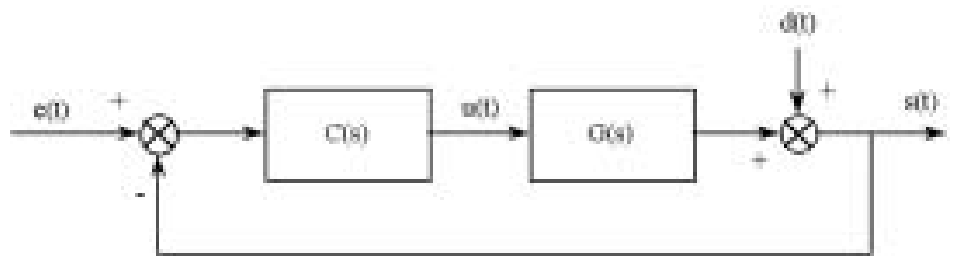

Figure 5. Control diagram

\subsection{Frequency Dynamic PERFormanCES}

In tracking, gain reaches a maximum for resonance frequency

$$
\omega_{t}=\left(-\cos \alpha \frac{\pi}{2}\right)^{1 / \alpha} \omega_{u}
$$

and in regulation, gain reaches a maximum for resonance frequency

$$
\omega_{r}=\left(-\cos \alpha \frac{\pi}{2}\right)^{-1 / \alpha} \omega_{u}
$$

This result reveals the existence of a resonance when $\cos (\alpha \pi / 2)<0$, namely for $1<\alpha<3$ and therefore for the CRONE control since $1<\alpha<2$.

The resonance ratio in tracking is expressed by the relation

$$
Q_{t}(\alpha)=\frac{\left|\mathbb{T}\left(j \omega_{t}\right)\right|}{|\mathbb{T}(j 0)|}=\frac{1}{\sin \alpha \frac{\pi}{2}},
$$

and the resonance ratio in regulation is expressed by

$$
Q_{r}(\alpha)=\frac{\left|\mathbb{S}\left(j \omega_{r}\right)\right|}{|\mathbb{S}(j \infty)|}=\frac{1}{\sin \alpha \frac{\pi}{2}} .
$$

These results show that the resonance ratio depends exclusively on control order $\alpha$, thus allowing the introduction of the notion of robust resonance.

Using (4.5) to (4.8) permits to define the link between the frequency dynamic performances in tracking and in regulation, namely :

$$
\left(\omega_{t} \omega_{r}\right)^{1 / 2}=\omega_{u}
$$

and

$$
Q_{t}(\alpha)=Q_{r}(\alpha)
$$

Relation (4.9) express that the resonance frequencies in tracking and in regulation are symmetrically distributed with regard to the open loop unit gain frequency.

Relation (4.10) expresses that the resonance ratios in tracking and in regulation are identical. Their identity is illustrated quite simply by the tangency of the vertical template to two amplitude contours with equal graduation, one the Nichols locus and the other the Oustaloup locus [19].

\subsection{Open loop transfer including the template}

The aim here is an analytical description of the open loop behavior (for the nominal plant) which takes into account:

- the accuracy specifications at low frequencies ; 
- the vertical template around unit gain frequency $\omega_{u}$;

- the input sensitivity specifications at high frequencies.

For a stable minimum phase plant, it turns out that the behavior thus defined can be described by a transmittance based on the frequency-limited real non integer differentiation, namely :

$$
\beta(s)=\left[K_{b}\left(\frac{\omega_{b}}{1}+1\right)\right]^{n_{b}}\left(\left(\frac{1+\left(\frac{\omega_{u}}{\omega_{b}}\right)^{2}}{1+\left(\frac{\omega_{u}}{\omega_{h}}\right)^{2}}\right)^{1 / 2} \frac{1+\frac{s}{\omega_{h}}}{1+\frac{s}{\omega_{b}}}\right)^{\alpha}\left(\frac{K_{h}}{1+\frac{s}{\omega_{h}}}\right)^{n_{h}}
$$

with :

$$
K_{b}=\left(1+\left(\frac{\omega_{b}}{\omega_{u}}\right)^{2}\right)^{-1 / 2} \quad \text { and } \quad K_{h}=\left(1+\left(\frac{\omega_{u}}{\omega_{h}}\right)^{2}\right)^{1 / 2}
$$

In the particular case where transitional frequencies $\omega_{b}$ and $\omega_{h}$ are sufficiently distant from frequency $\omega_{u}$, around this frequency (i.e. $\omega_{b}<<\omega<<\omega_{h}$ ), $\beta(s)$ can be reduced to transmittance

$$
\beta(s)=\left(\frac{\omega_{u}}{s}\right)^{\alpha},
$$

which is the same as that described by the template (relation (4.2)).

The order $\alpha$ transmittance of relation (4.11) describes the frequency truncation of the template defined by the transitional frequencies $\omega_{b}$ and $\omega_{h}$ [17]. This transmittance results from the substitution of the part raised at power $\alpha$ for the transmittance $\omega_{b} / p$ which is used in the description of the template between frequencies $\omega_{A}$ and $\omega_{B}$

If $n_{p b}$ represents the order of the asymptotic behavior of the plant in the low frequency domain $\left(\omega<\omega_{b}\right)$, order $n_{b}$ of the proportional-integrator is defined by :

$$
n_{b} \geqslant 1 \quad \text { if } \quad n_{p b}=0 \quad \text { and by } \quad n_{b} \geqslant n_{p b} \quad \text { if } \quad n_{p b} \geqslant 1 \text {, }
$$

given that $n_{b}=1$ cancels the position error and that $n_{b}=2$ cancels the hauling error [4]. Finally $K_{b}$ ensures unitary gain to the proportional integrator at frequency $\omega_{u}$.

If $n_{p h}$ denotes the order of the asymptotic behavior of the plant in the high frequency domain $\left(\omega>\omega_{h}\right)$, the low-pass filter order $n_{h}$ is defined by :

$$
n_{h} \geqslant n_{p h},
$$

given that (for $\omega>\omega_{h}$ ) $n_{h}=n_{p h}$ ensures constancy of the sensitivity of the input when the frequency rises, and $n_{h}>n_{p h}$ ensures its decrease as the frequency rises [13]. Finally $K_{h}$ ensures unitary gain to the low-pass filter at frequency $\omega_{u}$.

The order $\alpha$ of the template is determined by the tangency of the template at a given magnitude contour of the Nichols chart and the corresponding resonance ratio in tracking defines the first normalized overshoot of the free or step response in closed loop.

As frequency $\omega_{u}$ determines the rapidity of the dynamics of the closed loop, it is fixed by the designer to suit the input sensitivity $R(\omega)$ constraint defined by (4.17).

The high transitional frequency $\omega_{h}$ which determines the length of the template on the high frequency side is limited by a constraint which limits the sensitivity of the input, namely 


$$
\underset{G}{\operatorname{Sup}}(R(\omega))<R_{\max }
$$

with (fig. 5) :

$$
R(\omega)=\left|\frac{U(j \omega)}{E(j \omega)}\right|_{d(t)=0}=\left|\frac{U(j \omega)}{D(j \omega)}\right|_{e(t)=0}=\left|\frac{C(j \omega)}{1+\beta(j \omega)}\right|_{d(t)=0},
$$

where

$$
\beta(j \omega)=C(j \omega) G(j \omega) .
$$

The low transitional frequency $\omega_{b}$ which determines the length of the template on the low frequency side, can be chosen in accordance with the relation $\omega_{u}=$ $\left(\omega_{b} \omega_{h}\right)^{1 / 2}$ which adds no constraint to the control.

\subsection{Controller structure and parametric synthesis}

Controller $C(s)$ in cascade with the plant is synthesized from its frequency response deduced from the ratio:

$$
C(j \omega)=\frac{\beta(j \omega)}{G_{0}(j \omega)},
$$

where $G_{0}(j \omega)$ denotes the frequency response of the nominal plant.

Two techniques can be used for the synthesis of the CRONE controller $C(s)$. The first is based on the elementary symmetrical functions of Viete roots [20]. The second is based on the solving of a linear programming problem [21].

\section{The CRONE suspension}

\subsection{INTRODUCTION}

Two groups of factors influence the design of vehicle suspensions. The main factor of the first group is the vibration isolation requirement. This implies that over a wide range of operating conditions the suspension must provide a comfortable ride for passengers. In the second group, one of the most important factors is the stroke length design constraint. It represents the maximal allowable relative displacement between the bodywork and wheels. Additional constraints are imposed by the stability, safety and handling requirements of the vehicle [22]. Finally, there are always cost constraints.

Passive, semi-active and active suspensions satisfy these requirements to differing degrees [23]. Passive suspensions are characterized by the absence of any controlled energy sources. For this reason they are relatively inexpensive and reliable. Active suspensions require energy sources such as compressors or pumps. Semi-active suspensions, as their name implies, fill the gap between purely passive and purely active suspensions. The improved performances of active and semi-active suspensions implies increased hardware complexity, higher costs and diminished reliability.

In this paper, a new passive suspension based on non integer differentiation is presented. The principle is to replace the spring and damper of a traditional suspension by a mechanical system defined by a non integer order force-displacement transmittance. The suspension thus obtained is called the CRONE suspension because of the link with the second generation CRONE control through the vertical template [13]. This is why the principle of the second generation CRONE control is used to synthesize suspension transmittance. Suspension parameters are then 
determined from a constrained optimization of a performance criterion. The frequency and time responses, for various values of the vehicle load, reveal a great robustness of the stability degree through the constancy of the resonance ratio in the frequency domain, and of the damping ratio in the time domain.

From the concept of the CRONE suspension several technological solutions have been developed [24]. One of them, called passive CRONE suspension, uses the link between recursivity and non integer differentiation.

\subsection{VEHICLE MODEL}

The suspension system, located between the bodywork and wheel, develops a force $f(t)$ which can be generated by an active, semi-active or passive device [25] [26]. For example, a traditional suspension (fig. 6.a) develops a force $f(t)$ which is a function of the relative displacement $\left(z_{u}(t)-z_{s}(t)\right)$ and given by :

$$
f(t)=k\left(z_{u}(t)-z_{s}(t)\right)+b \frac{d}{d t}\left(z_{u}(t)-z_{s}(t)\right),
$$

in which $z_{u}(t)$ and $z_{s}(t)$ are the vertical displacements of the wheel (unsprung mass) and bodywork (sprung mass), $k$ the stiffness of the spring and $b$ the damping coefficient.

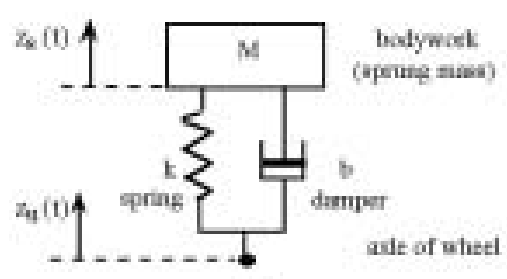

(a)

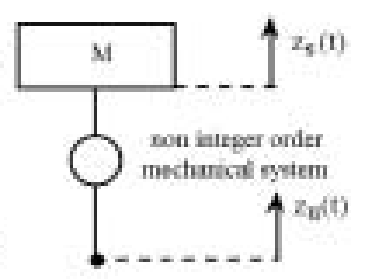

(b)

Figure 6. Quarter car model with traditional (a) and CRONE (b) suspensions

The CRONE suspension develops a force $f(t)$ which is a function of the relative displacement and which obeys symbolically the general relation :

$$
F(s)=C(s)\left(Z_{u}(s)-Z_{s}(s)\right),
$$

in which $C(s)$ is the suspension transmittance defined by a non integer expression (5.13).

If it is assumed that the tyre does not leave the ground and that $z_{u}(t)$ and $z_{s}(t)$ are measured from the static equilibrium position, then the application of the fundamental law of dynamics leads to the linearized equation of motion :

$$
M\left(\frac{d}{d t}\right)^{2} z_{s}(t)=f(t),
$$

in which $M$ is the sprung mass. The Laplace transform of equation (5.3), assuming zero initial conditions, is

$$
M s^{2} Z_{s}(s)=F(s) \text {. }
$$

To analyze the vibration isolation of the sprung mass, two transmittances are defined :

ESAIM: ProC., VOL. 5, 1998, 177-192 


$$
T(s)=\frac{Z_{s}(s)}{Z_{u}(s)} \quad \text { and } \quad S(s)=\frac{Z_{u}(s)-Z_{s}(s)}{Z_{u}(s)}=1-T(s) .
$$

From equations (5.1), (5.2) and (5.4), the expressions of $T(s)$ and $S(s)$ are given by :

$$
T(s)=\frac{k+b s}{k+b s+M s^{2}} \quad \text { and } \quad S(s)=\frac{M s^{2}}{k+b s+M s^{2}}
$$

for the traditional suspension,

$$
T(s)=\frac{C(s)}{C(s)+M s^{2}} \quad \text { and } \quad S(s)=\frac{M s^{2}}{C(s)+M s^{2}}
$$

for the CRONE suspension.

\subsection{Synthesis of suspension transmittance}

The synthesis method of the CRONE suspension is based on transmittances $T(s)$ and $S(s)$ which can be written as :

$$
T(s)=\frac{\beta(s)}{1+\beta(s)} \quad \text { and } \quad S(s)=\frac{1}{1+\beta(s)},
$$

in which :

$$
\beta(s)=\frac{C(s)}{M s^{2}} .
$$

The transmittances $T(s)$ and $S(s)$ can here be considered to be these of an elementary control loop whose $\beta(s)$ is the open loop transmittance.

Given that relation (5.9) expresses that a variation of mass is accompanied by a variation of open loop gain, the principle of the second generation CRONE control can be used to synthesize the open loop Nichols locus which traces a vertical template for the nominal mass.

A way of synthesizing the open loop Nichols locus consists in determining a transmittance $\beta(s)$ which has successively (Fig. 7):

- an order 2 asymptotic behavior at low frequencies to eliminate tracking error

- an order $\alpha$ asymptotic behavior where $\alpha$ is between 1 and 2, exclusively around frequency $\omega_{u}$ to limit the synthesis of the non integer differentiation at a truncated frequency interval ;

- an order 1 asymptotic behavior at high frequencies, to ensure satisfactory filtering of vibrations at high frequencies.

Such a behavior can be obtained with a transmittance of the form :

$$
\beta(s)=C_{0} \frac{\left(1+\frac{s}{\omega_{b}}\right)^{\alpha^{\prime}}}{\left(1+\frac{s}{\omega h}\right)^{\alpha^{\prime}-1}}\left(\frac{\omega_{0}}{s}\right)^{2},
$$

in which

$$
\left.\omega_{b}<<\omega_{A}, \quad \omega_{B}<<\omega_{h} \quad \text { and } \quad \alpha^{\prime}=2-\alpha \in\right] 0,1[.
$$

Identification of equations (5.9) and (5.10) gives : 


$$
\frac{1}{\sqrt{M}}=\omega_{0}
$$

and

$$
C(s)=\frac{F(s)}{Z_{u}(s)-Z_{s}(s)}=C_{0} \frac{\left(1+\frac{s}{\omega_{b}}\right)^{\alpha^{\prime}}}{\left(1+\frac{s}{\omega_{h}}\right)^{\alpha^{\prime}-1}} .
$$

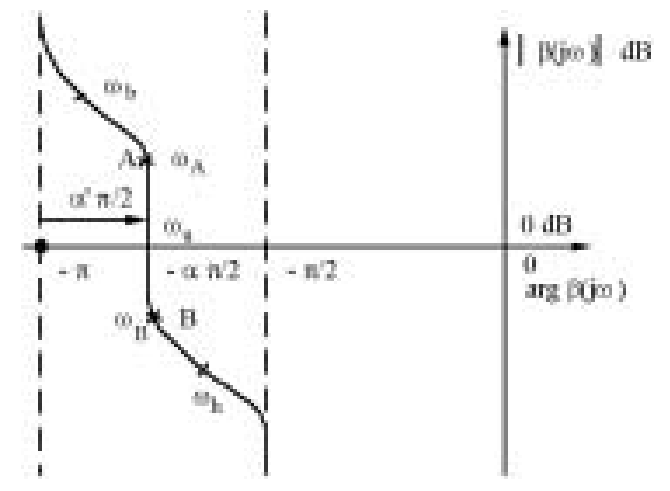

Figure 7. Open loop Nichols locus of the CRONE suspension

The equation thus obtained defines the ideal version of the suspension. The corresponding real version is defined by an integer order transmittance of the form [13] :

$$
C_{N}(s)=\frac{F(s)}{Z_{u}(s)-Z_{s}(s)}=C_{0} \frac{\prod_{i=1}^{N}\left(1+\frac{s}{\omega_{i}}\right)}{\prod_{i=1}^{N-1}\left(1+\frac{s}{\omega_{i}}\right)}
$$

in which

$$
\begin{gathered}
\frac{\omega_{i+1}^{\prime}}{\omega_{i}^{\prime}}=\frac{\omega_{i+1}}{\omega_{i}}=\gamma \eta>1 ; \quad \frac{\omega_{i}}{\omega_{i}^{\prime}}=\gamma ; \quad \frac{\omega_{i+1}^{\prime}}{\omega_{i}}=\eta ; \quad \gamma \eta=\left(\frac{\omega_{h}}{\omega_{b}}\right)^{\frac{1}{N}} ; \\
\gamma=(\gamma \eta)^{\alpha^{\prime}} ; \quad \omega_{1}^{\prime}=\omega_{b} \eta^{\frac{1}{2}} \quad \text { and } \quad \omega_{N}=\omega_{h} \eta^{\frac{-1}{2}}
\end{gathered}
$$

\subsection{Performance}

The design of advanced vehicle suspension can be formulated as an optimal control problem. The main objective of the suspension is to provide good vibration isolation for comfort and to maintain adequate adherence of the wheels for braking, accelerating and handling. Additional constraints limit suspension stroke. The objective and the constraints can be formulated in an optimality criterion [24]. In addition, to obtain a significant comparison between traditional and CRONE suspension performances, a constraint is fixed for the minimal sprung mass : equal unit gain frequency of open loop $\beta(j \omega)$.

The traditional suspension used for comparison is the rear hydropneumatic suspension of a Citroën BX. The parameters of the quarter-car model are given by 
- sprung mass : $150 \mathrm{~kg} \leq M \leq 300 \mathrm{~kg}$;

- stiffness : $k=2500 \mathrm{~N} / \mathrm{m}$;

- damping coefficient : $b=850 \mathrm{Ns} / \mathrm{m}$.

From this data, the constrained optimization, computed using the optimization toolbox of Matlab (c), provides the optimal parameters of the CRONE suspension, namely :

- for the ideal version :

$$
\alpha^{\prime}=0.8 ; \quad C_{0}=215 ; \quad \omega_{b}=0.1 r d / s ; \quad \omega_{h}=200 r d / s ;
$$

- for the real version :

$$
N=5 ; \quad C_{0}=215 ; \quad \gamma=\frac{\omega_{i}}{\omega_{i}^{\prime}}=3.37 ; \quad \eta=\frac{\omega_{i+1}^{\prime}}{\omega_{i}}=1.355 ; \quad \omega_{1}^{\prime}=0.1164 r d / s
$$

5.4.1. Frequency Responses. Fig. 8 and 9 show the frequency performances in open loop and closed loop.

Fig. 8 gives the Nichols loci of $\beta(j \omega)$ for the traditional and CRONE suspensions. The phase margin varies with mass $M$ for the traditional suspension. On the other hand, the phase margin is independent for the CRONE suspension, where the Nichols loci in open loop trace the template which characterizes the second generation CRONE control.

Fig. 9 gives the gain diagrams of $T(j \omega)$ for the traditional and CRONE suspensions. For the CRONE suspension, the resonance ratio can be seen to be both weak and insensitive to variations of mass $M$. This shows a better robustness of the CRONE suspension in the frequency domain.

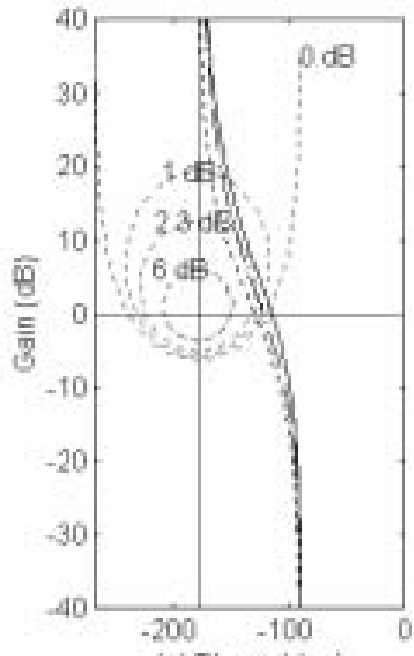

(a) Phase (deg)

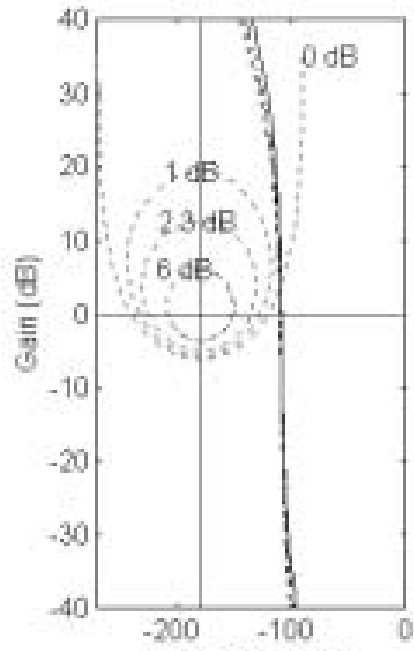

(b) Phise (deg)

Figure 8. Nichols loci in open loop for traditional (a) and CRONE (b) suspension : $-M=150 \mathrm{~kg} ;--M=225 \mathrm{~kg} ; \ldots . . M=$ $300 \mathrm{~kg}$ 

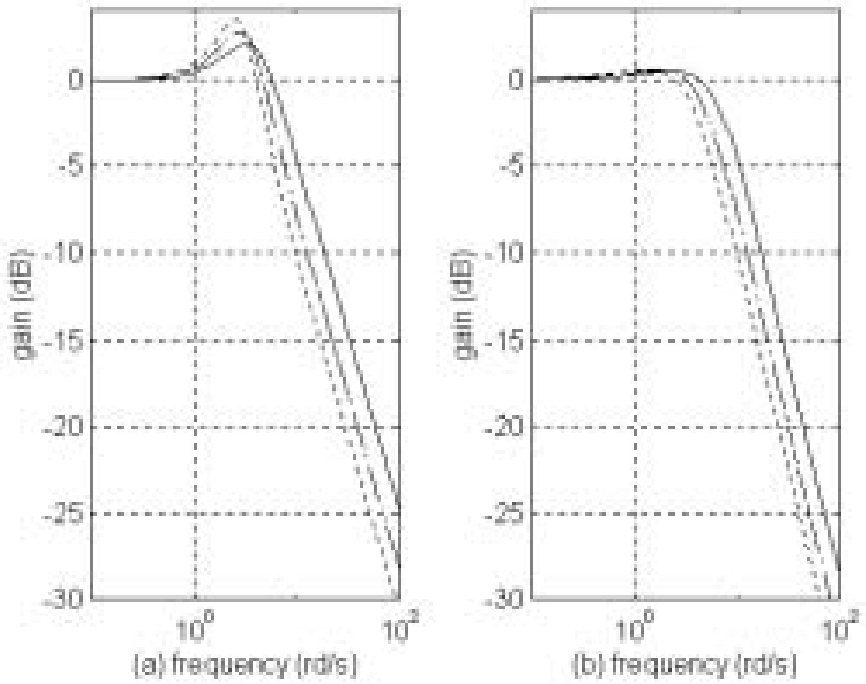

Figure 9. Gain diagrams of $T(j \omega)$ for traditional (a) and CRONE (b) suspension : $-M=150 \mathrm{~kg}$; - $M=225 \mathrm{~kg} ; \ldots . M=$ $300 \mathrm{~kg}$

5.4.2. Step Responses. Fig. 10 gives the step responses of the bodywork for both suspensions. For the CRONE suspension it can be seen that the first overshoot remains constant, showing a better robustness for the CRONE suspension in the time domain (Fig. 10.b).

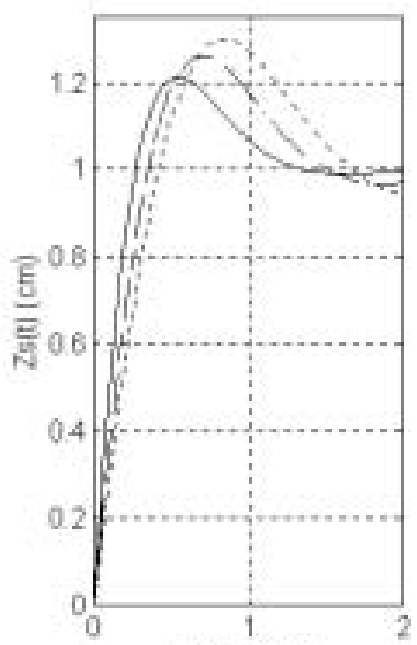

(a) time (s)

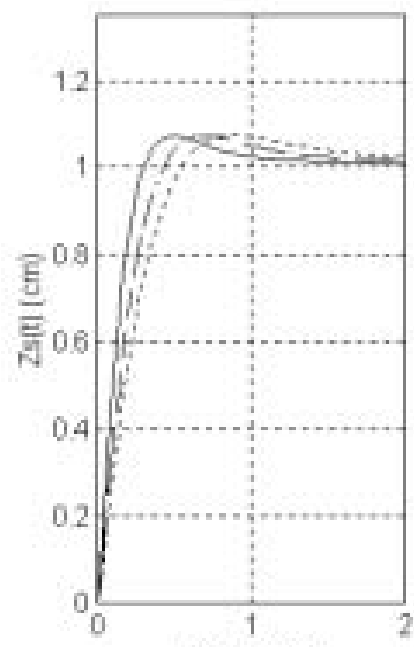

(b) time (s)

FiguRE 10. Step responses of sprung mass for traditional (a) and CRONE (b) suspension : $-M=150 \mathrm{~kg}$; - $M=225 \mathrm{~kg} ; \ldots . M=$ $300 \mathrm{~kg}$ 


\section{Conclusion}

In this paper, a physical phenomenon have been used to develop a robust control strategy based on real non integer differentiation. This study has allowed recent developments which lead to the replacement of the vertical template, either by a generalized template defined as a straight line segment of any direction, or by a multi-template defined as a set of templates of a similar type. Real non integer integration used in the description of the vertical template is in this case replaced by complex non integer integration. The control is then robust to reparametrations of the plant which does not necessarily lead to variations of gain around the open loop unit gain frequency. The generalization thus defined is the object of the third generation CRONE control [4] which can be applied to multivariable or time-varying plants. Note that other control strategies based on non integer approaches [28], [27], sometimes closely related to CRONE principles [15], can be found in the literature.

The study of the CRONE suspension has also shown the usefulness of non integer differentiation in vibration isolation. From the concept of the CRONE suspension, several technological solutions have been developed [24]. One of them, called passive CRONE suspension is based on a recursive distribution of springs and dampers. This suspension has been implemented on an experimental Citroën BX. Bench and road tests on this prototype have validated theoretical expectations [24]. Other technological solutions based on energetical transfer have also been studied. These technological solutions are called active and passive piloted CRONE suspension and are actually tested. New versions of passive CRONE suspension based on viscoelastic fluid flow in porous media are also studied [14].

\section{REFERENCES}

[1] A. Oustaloup and J. Sabatier - Study of the relaxation of water on a porous dyke : a non integer approach - Annales Geophysicae, Vol. 13 - 1995.

[2] A. Oustaloup, X. Moreau and M. Nouillant - The CRONE suspension - Control Engineering Practice, a Journal of the International Federation of Automatic Control - Vol 4, $\mathrm{N}^{\circ} 8$, pp. 1101-1108, 1996.

[3] R. W. Glenny and H. T. Robertson - Fractal properties of pulmonary blood flow : characterization of spatial heterogeneity - American Physiological Society - 1990.

[4] F. de Carfort, C. Foulard et J. Calvet - Asservissements linéaires continus - Dunod Université $-1976$.

[5] A. Oustaloup, P. Lanusse et B. Mathieu - Robust control of SISO plants : the CRONE control - Proceedings of the European Control Conference - p 1423 - Rome - 1995.

[6] B. Mandelbrot, The fractal geometry of nature, Freeman, San Francisco (USA), 1982.

$[7]$ K. B. Oldham and J. Spanier - The fractional calculus - Academic Press - New York and London - 1974.

[8] F. Nanot, A. Oustaloup, L. Le Lay and F. Levron - Generalized differential system : application to the multiscalar CRONE control of a cutting table - IEEE-SMC'96, IMACS Multiconference - Symposium on Control, Optimization and Supervision - Lille, july 9-12 1996.

[9] A. Le Mehaute and G. Crepy, Ionics 9\&10, North-Holland Publishing Company, 1983.

[10] G. Montseny, J.Audounet and B. Mbodje - Optimal models of fractional integrators and application to systems with fading memory - IEEE SMC's confererence, Le Touquet - 17-20 oct 1993 - pp 65-70.

[11] P. Cosson et J. C. Michon - Identification par modèle non entier du comportement mécanique d'un élastomère - Journées d'étude "les systèmes d'ordre non entier en automatique" - GDR automatique - Bordeaux 25-26 Avril 1995.

[12] D. Matignon, B. d'Andréa Novel, P. Depalle and A. Oustaloup - Viscothermal losses in wind instrument : a non-integer model - Systems and networks : mathématical theory and application - Vol II, volume 79 - p 789-790 - Akademic Verlag - 1994

[13] A. Oustaloup - La commande CRONE : Commande robuste d'ordre non entier - Editions Hermes - 1991. 
[14] M. Nouillant, P. David, X. Moreau and A. Oustaloup - A new hydropneumatic suspension system using a porous media and a viscoelactic fluid : the passive CRONE suspension Advances in Vehicle Control and Safety AVCS'98 - Amiens - July 1998.

[15] G. Montseny, J. Audounet and J. Bernussou - Diffusive approach to robust control - IFAC conference on Systems, Structure and Control - Vol. 2 - pp243-248 - Nantes - 1998.

[16] A. Oustaloup, La dérivation non entière : théorie, synthèse et application dans les sciences pour l'ingénieur, Editions Hermès, 1995.

[17] A. Oustaloup, F. Levron, F. Nanot and B. Mathieu - Frequency band complex non integer differentiator : characterisation and synthesis - IEEE transactions on Circuit and Systems to appear - 1998.

[18] A. Oustaloup and M. Nouillant - Distributed parameter systems and non integer differentiation - session "Robust control design in frequency domain" - ECC'91 Grenoble 1991.

[19] A. Oustaloup - Etude de la fonction régulation d'un système asservi au moyen d'un nouvel abaque - L'onde électrique, 1978 , vol. $58, \mathrm{n}^{\circ} 8-9$ pp.543-546.

[20] A. Oustaloup, B. Mathieu and P. Lanusse - The CRONE control of resonant plants : application to a flexible transmission - European Journal of Control - Vol. 1 - $\mathrm{N}^{\circ} 2$ - pp113-121 1995.

[21] B. Mathieu, A. Oustaloup et F. Levron - Transfer function parameter estimation by interpolation in the frequency domain - European control conference ECC'95 - Vol 3 - p 2131 Rome 1995.

[22] X. Moreau, A. Oustaloup and M. Nouillant, "Comparison of LQ and CRONE methods for the design of suspension systems", 13th IFAC World Congress, San Francisco, USA, 1996.

[23] Hrovat D., Margolis D. L. and Hubbard M, "An approach toward the optimal semi-active suspension", Journal of Dynamic Systems, Measurement and Control, Vol. 110, 1988, pp.288296.

[24] Moreau X, "Non-integer differentiation in vibration isolation and its application in automobiles. The CRONE suspension: from design concept to prototype", Ph.D thesis, University of Bor-deaux I, 1995.

[25] Yue C., Butsuen T. and Hedrick J. K, "Alternative control laws for automotive active suspensions", Journal of Dynamic Systems, Mea-surement and Con-trol, Vol. 111, 1989, pp. 286-291.

[26] Sharp R. S. and Hassan S. A, "The relative performance capabilities of passive, active and semi-active car suspension systems", Proc. Inst. Mech. Engers., vol. 200, 1986, pp. 219-228.

[27] G. Montseny, J. Audounet, D. Matignon, "Fractional integrodifferential boundary control of the Euler-Bernoulli beam", 36th IEEE CDC's conference, San Diego (USA), 1997, pp 4973-4978.

[28] D. Matignon, "Stability results for fractional differential equations with applications to control processing", Computational Engineering in Systems and Application multiconference, vol. 2, pages 963-968, IMACS, IEEE-SMC. Lille, France, July 1996. 